\title{
Anticipating the Response of Excitable Systems Driven by Random Forcing
}

\author{
M. Ciszak, ${ }^{1}$ O. Calvo, ${ }^{1}$ C. Masoller, ${ }^{1,3}$ Claudio R. Mirasso, ${ }^{1}$ and R. Toral ${ }^{1,2}$ \\ ${ }^{1}$ Departament de Física, Universitat de les Illes Balears, E-07122 Palma de Mallorca, Spain \\ ${ }^{2}$ Instituto Mediterraneo de Estudios Avanzados, CSIC-UIB, Edificio Mateu Orfila, Campus UIB, \\ E-07122 Palma de Mallorca, Spain \\ ${ }^{3}$ Instituto de Física, Facultad de Ciencias, Universidad de la República, Igua 4225, Montevideo 11400, Uruguay
}

(Received 17 September 2002; published 22 May 2003)

\begin{abstract}
We study the regime of anticipated synchronization in unidirectionally coupled model neurons subject to a common external aperiodic forcing that makes their behavior unpredictable. We show numerically and by analog hardware electronic circuits that, under appropriate coupling conditions, the pulses fired by the slave neuron anticipate (i.e., predict) the pulses fired by the master neuron. This anticipated synchronization occurs even when the common external forcing is white noise.
\end{abstract}

DOI: $10.1103 /$ PhysRevLett.90.204102

PACS numbers: 05.40.Ca, 42.65.Pc, 42.65.Sf

Synchronization of nonlinear systems is a fascinating subject that has been extensively studied on a large variety of physical and biological systems. While synchronization of oscillators goes back to the work by Huygens, the past decade has witnessed an increased interest in the topic of synchronization of chaotic systems [1].

Recently, Voss [2] discovered a new scheme of synchronization, called "anticipated synchronization." Voss showed that by using appropriate delay lines it is possible to synchronize two unidirectionally coupled systems in such a way that the slave system, $\mathbf{y}(t)$, predicts the behavior of the master system, $\mathbf{x}(t)$. Two coupling schemes were considered: complete replacement,

$$
\begin{aligned}
& \dot{\mathbf{x}}(t)=-\alpha \mathbf{x}(t)+\mathbf{f}(\mathbf{x}(t-\tau)), \\
& \dot{\mathbf{y}}(t)=-\alpha \mathbf{y}(t)+\mathbf{f}(\mathbf{x}(t)),
\end{aligned}
$$

and delay coupling,

$$
\dot{\mathbf{x}}(t)=\mathbf{f}(\mathbf{x}(t)), \quad \dot{\mathbf{y}}(t)=\mathbf{f}(\mathbf{y}(t))+\mathbf{K}[\mathbf{x}(t)-\mathbf{y}(t-\tau)] .
$$

$\mathbf{f}(\mathbf{x})$ is a function which defines the autonomous dynamical system under consideration. It is easy to see that in both schemes the manifold $\mathbf{y}(t)=\mathbf{x}(t+\tau)$ is a solution of the equations, and Voss has shown that in both schemes this solution can be structurally stable. This is more remarkable when the dynamics of the master system $\mathbf{x}$ is "intrinsically unpredictable," as is the case of a chaotic system. While in the scheme of complete replacement the anticipation time $\tau$ can be arbitrarily large, the delay coupling scheme requires some constraints on the anticipation time $\tau$ and coupling $\mathbf{K}$ for the synchronization solution to be stable [2]. Despite this fact, the delay coupling scheme is more interesting since the anticipation time $\tau$ can be varied without altering the dynamics of the master system $\mathbf{x}$.

In this Letter, we study numerically and experimentally the regime of anticipated synchronization in excitable nonautonomous systems. In our case, the intrinsic unpredictability of the behavior of the dynamical system $\mathbf{x}$ does not arise from a chaotic dynamics, but rather from the existence of an external forcing with some element of randomness. We consider the coupled systems

$$
\begin{aligned}
\dot{\mathbf{x}}(t) & =\mathbf{f}(\mathbf{x}(t))+\mathbf{I}(t), \\
\dot{\mathbf{y}}(t) & =\mathbf{f}(\mathbf{y}(t))+\mathbf{I}(t)+\mathbf{K}[\mathbf{x}(t)-\mathbf{y}(t-\tau)],
\end{aligned}
$$

where $\mathbf{I}(t)$ represents the common external forcing. Notice that $\mathbf{y}(t)=\mathbf{x}(t+\tau)$ is no longer an exact solution of the equations [except in the particular case of a periodic forcing $\mathbf{I}(t+\tau)=\mathbf{I}(t)]$. We show that under appropriate coupling conditions there can be a very good correlation between $\mathbf{y}(t)$ and $\mathbf{x}(t+\tau)$ which, in practice, allows the prediction of the future behavior of $\mathbf{x}(t)$ with a high degree of accuracy.

Specifically, we have considered models of sensory neurons. Sensory neurons transform external stimuli signals as pressure, temperature, electric pulses, etc., into trains of action potentials, usually referred to as "spikes" or "firings." Their behavior is typical of excitable systems: If the forcing is above a certain threshold, the neuron fires a pulse and, after the firing, the recovery process produces an absolute refractory time during which a second firing cannot occur. In general, sensory neurons work in a noisy environment. As a consequence, the time intervals between spikes contain a significant random component, and random spikes often occur even in the absence of stimuli. The topics of synchronous oscillations and noise have received much attention (see, e.g., [3]), since it has been pointed out that synchronous firing activity of sensory neurons might be a part of higher brain functions and a method for integrating distributed information into a global picture [4].

Here we show that the interplay of coupling, delayed feedback, and common noise can lead to anticipated synchronization. We illustrate this effect in the wellknown FitzHugh-Nagumo and Hodkey-Huxley neuron models. By coupling two such systems in a unidirectional 
configuration, we find that, under appropriate conditions and when both systems are subjected to the same external random forcing, the slave system fires the same train of spikes as the master system, but at a certain amount of time earlier; i.e., the slave system predicts the response of the master system.

First we show results based on the FitzHugh-Nagumo model consisting of two variables $\mathbf{x}=\left(x_{1}, x_{2}\right)$. The fast variable, $x_{1}$, is associated with the activator, and the slow recovery variable, $x_{2}$, is associated with the inhibitor. The equations for the master $\left(x_{1}, x_{2}\right)$ and the slave $\mathbf{y}=\left(y_{1}, y_{2}\right)$ systems under unidirectional coupling are, respectively (see the schematic diagram shown in Fig. 1),

$$
\begin{aligned}
& \dot{x}_{1}=-x_{1}\left(x_{1}-a\right)\left(x_{1}-1\right)-x_{2}+I(t), \\
& \dot{x}_{2}=\epsilon\left(x_{1}-b x_{2}\right),
\end{aligned}
$$

and

$$
\begin{aligned}
\dot{y}_{1}= & -y_{1}\left(y_{1}-a\right)\left(y_{1}-1\right)-y_{2}+I(t) \\
& +K\left[x_{1}(t)-y_{1}(t-\tau)\right], \\
\dot{y}_{2}= & \epsilon\left(y_{1}-b y_{2}\right),
\end{aligned}
$$

where $a, b$, and $\epsilon$ are constants. The parameter $K$ controls the strength of the coupling and $\tau$ is a delay time associated to an inhibitory feedback loop in the slave neuron.

We have considered different types of random external forcing $I(t)$. The first one corresponds to a random process whose amplitude remains constant for a time $T$ but then it switches to a new value chosen uniformly in $\left(I_{0}-D\right.$, $I_{0}+D$ ), where $D$ is the noise intensity. We chose $I_{0}$ very close to, but below, the firing threshold of the excitable system. It would appear at first thought that with this external forcing the behavior of the master system can be easily predicted: If $I(t)>I_{0}$, the system fires a pulse; otherwise it does not. However, we should keep in mind that the system has a refractory time (after firing a pulse) during which another firing is not possible. Therefore, even if the external forcing is rather simple, the behavior of the master system is unpredictable. Figure 2 shows that anticipation occurs with this type of random external forcing for an appropriate value of the coupling strength $K$ : After an initial transient time, the two systems syn-

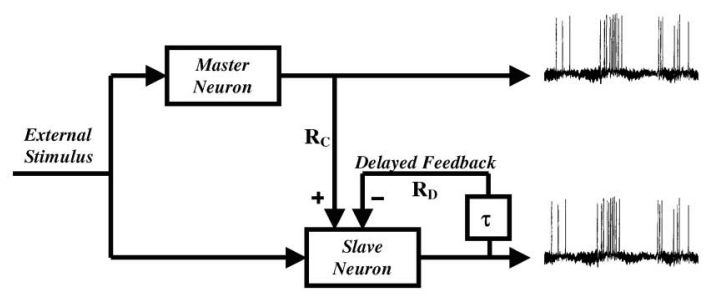

FIG. 1. Schematic diagram of two model neurons coupled in a unidirectional configuration, subjected to the same external forcing and with a feedback loop (with a delay time $\tau$ ) in the slave neuron. chronize such that the slave system anticipates the fires of the master system by a time interval $\tau$. The firings in the master and the slave systems start at about the same time, and the anticipation phenomenon grows during the rising of the pulse. When the master system noisily evolves near the stable point, the anticipation vanishes. In other words, anticipation is a local process, during firings.

The same qualitative results are found with other types of external forcing such as colored or even white noise. [Figures 3(a) and 3(b) display the spikes of the master and slave systems when $I(t)$ is a Gaussian white noise.] Note that the systems for which anticipated synchronization has been studied thus far are low-dimensional chaotic systems, while the behavior of our systems under the effect of noise can be considered to have a much higher degree of unpredictability than that of any chaotic system.

Sometimes the slave system makes an error in anticipating the master firings. While the slave system always fires a pulse when the master system fires a pulse, it also might fire an "extra" pulse, which has no corresponding pulse in the train of pulses fired by the master. Notice that in Fig. 3(a) an error at about $t=1900$ occurs. Not surprisingly, we find that the longer the anticipation time $\tau$, the larger the number of errors. However, for a given anticipation time, the number of errors can be reduced considerably if a "cascade" of an adequate number of slave neurons is considered. A detailed study of the number of errors and its dependency with the type of external forcing will be reported elsewhere. Synchronization disappears for a very large value of the coupling constant. This counterintuitive behavior is in agreement with a linear analysis of the model that predicts that the maximum coupling for synchronization decays roughly as the inverse of the delay time $\tau$.

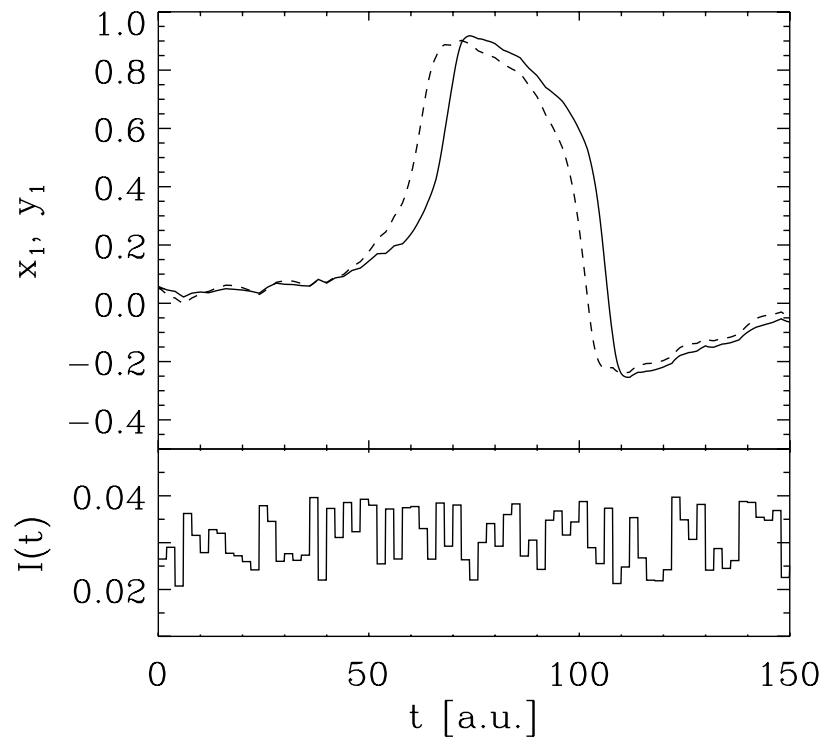

FIG. 2. Anticipated synchronization for random amplitude noise in the case of a FitzHugh-Nagumo. 

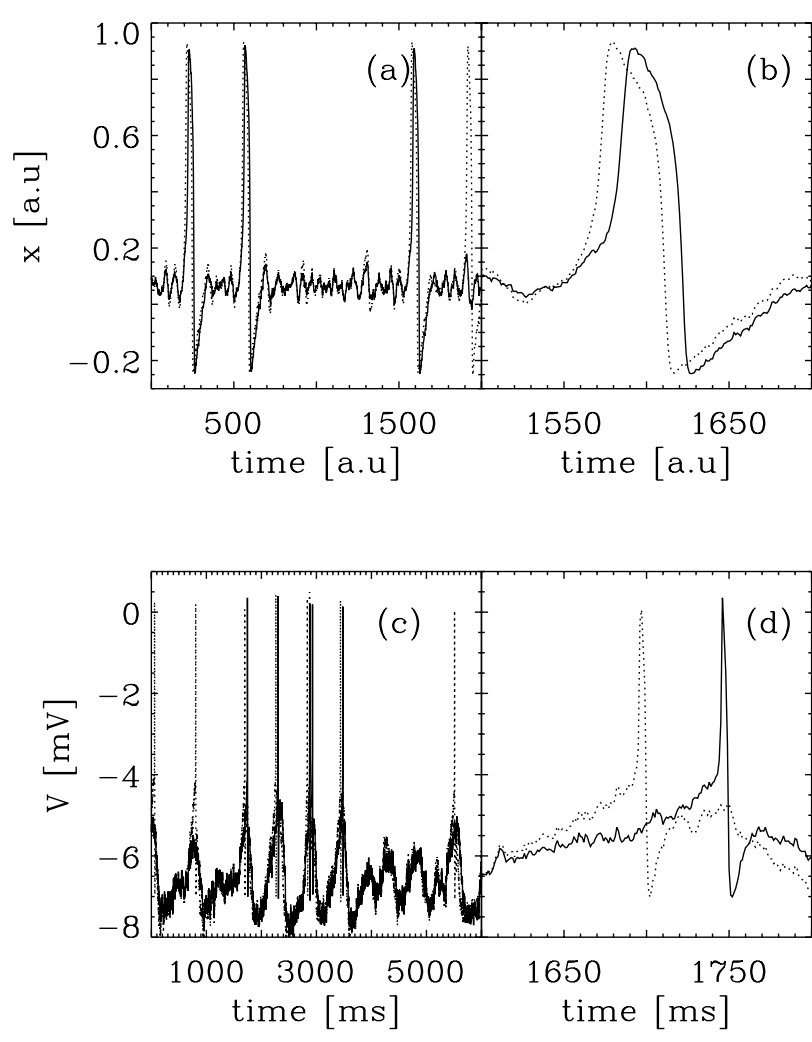

FIG. 3. Trains of spikes obtained from numerical simulations of models of unidirectionally coupled neurons subjected to the same external forcing, which is a Gaussian white noise with zero mean and correlation $\left\langle I(t) I\left(t^{\prime}\right)\right\rangle=2 D \delta\left(t-t^{\prime}\right)$. (a) Simulation of two FitzHugh-Nagumo neurons. The parameters are $a=0.139, b=2.54, \epsilon=0.008, I_{0}=0.03, K=0.03$, $\tau=10$, and $D=2.45 \times 10^{-5}$. (b) Simulation of two HodgkinHuxley neurons: $K=0.03 \mathrm{~ms}^{-1}, \tau=50 \mathrm{~ms}$, and $D=$ $0.5 \mathrm{mV}^{2} / \mathrm{ms}$. $T=6 \mathrm{C}, V_{l}=-75 \mathrm{~mA}$; all other parameters as in [5]. Left panels show typical spike trains; right panels show with detail a single spike. The solid (dashed) line represents the output of the master (slave) system.

Next we show simulations based on a more realistic model, namely, the model of electroreceptors proposed by Braun et al. [5]. This model is a modification of the Hodgkin-Huxley neuron model: $C_{M} \dot{x}=-i_{\mathrm{Na}}-i_{\mathrm{K}}-$ $i_{s d}-i_{s r}-i_{l}$, where $x$ is the potential voltage across the membrane and $C_{M}$ is the capacitance; $i_{\mathrm{Na}}$ and $i_{K}$ are the fast sodium and potassium currents; $i_{s d}$ and $i_{s r}$ are additional slow currents; $i_{l}$ is a passive leak current. For details and functional dependence of the currents on $x$, see [5].

We extend the model to account for two unidirectionally coupled neurons subject to a common external forcing $I(t)$, and with a delayed feedback loop in the slave neuron. This is done in the same way as in the FitzHughNagumo model, i.e., by including a term of the form $K[x(t)-y(t-\tau)]$ in the dynamics of the slave neuron. Figure 3(b) shows the results when the common external forcing is a Gaussian white noise. We chose parameters such that in the absence of forcing there are no spikes (subthreshold, noise-activated firing regime). The behavior observed is qualitatively the same as in the FitzHughNagumo model (the slave neuron anticipates the fires of the master neuron), which indicates that the anticipation phenomenon is general and model independent. Remarkably, in this model the anticipation time can be larger than the pulse duration. It is worth mentioning that anticipated synchronization is also observed for parameters such that there is spontaneous (regular or irregular) spike activity (suprathreshold firing regime).

To assess the robustness of the anticipated synchronization observed in the numerical simulations, we have implemented the FitzHugh-Nagumo model in analog hardware and constructed two coupled electronic neurons (a simplified version of the circuit is shown in Fig. 4). The electronic neurons were built using operational amplifiers and the cubic nonlinearity described by $x(x-a)(x-1)$ was implemented using analog multipliers (AD633) in a circuit not shown for simplicity. The resistor $R_{C}$ controls the strength of the unidirectional coupling between the master and the slave neurons. The resistor $R_{D}\left(R_{D}=R_{C}\right.$ in our case) controls the strength of the delayed feedback into the slave neuron. The coupling and the delayed feedback have opposite signs: While the master signal was obtained at point $B$ of Fig. 4, where the voltage is $-V_{m}$, the slave signal that goes into the delay line was obtained at point $C$, where the voltage is $+V_{s}$. The different signs are due to the inverters that are located in between points $A$ and $B$ and $C$ and $D$. The threshold on both neurons was controlled by a potentiometer represented by its equivalent circuit: offset and $R_{0}$. The analog delay line for the delayed feedback in the slave neuron was built using

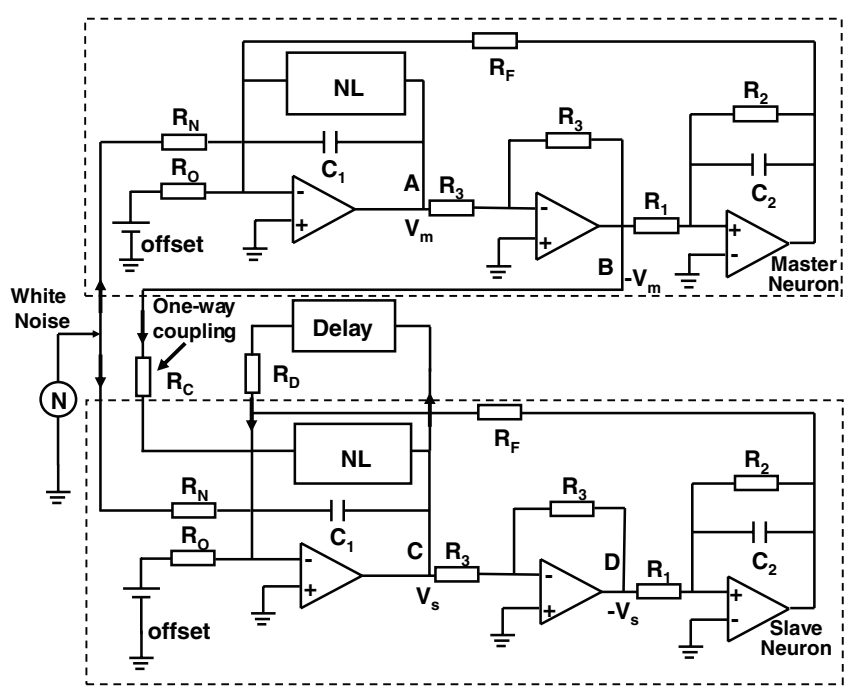

FIG. 4. Circuit implementation of two coupled neurons. $R_{1}=$ $125 \mathrm{k} \Omega, \quad R_{2}=50 \mathrm{k} \Omega, \quad R_{3}=10 \mathrm{k} \Omega, \quad R_{C}=R_{D}=100 \mathrm{k} \Omega$, $R_{F}=10 \mathrm{k} \Omega, R_{N}=10 \mathrm{k} \Omega, R_{O}=10 \mathrm{k} \Omega, C_{1}=100 \mathrm{nF}$, and $C_{2}=1 \mu \mathrm{F}$. 


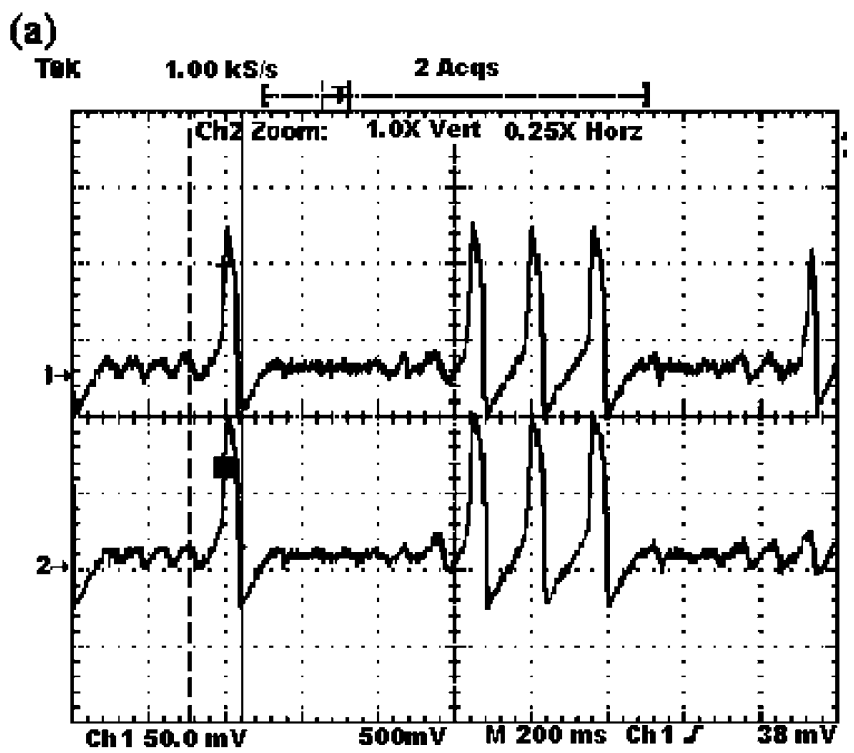

(b)

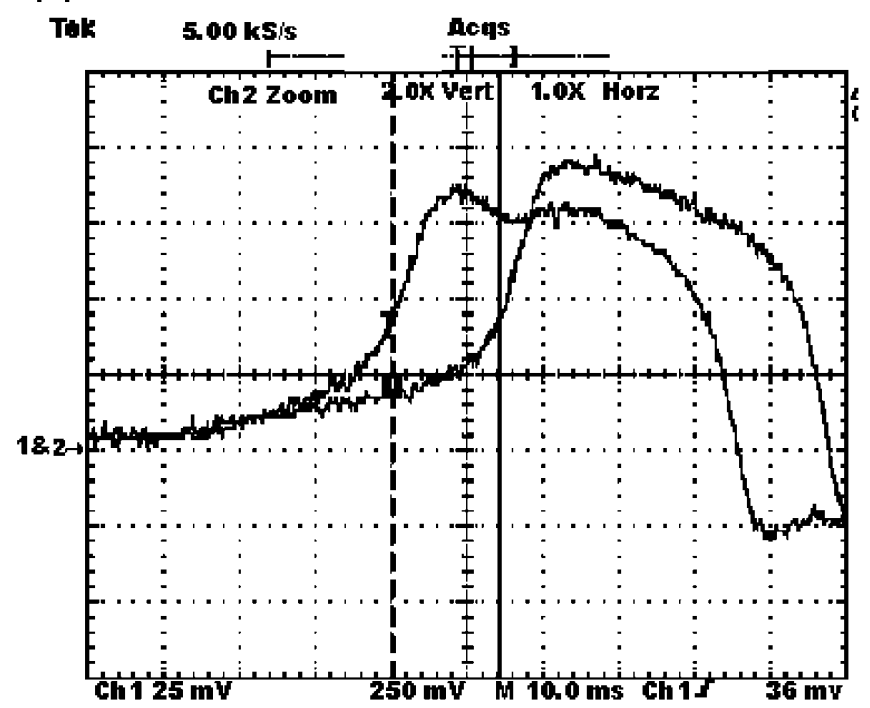

FIG. 5. (a) Experimental train of spikes that shows anticipation in the spikes fired by the slave neuron (upper trace) with respect to the spikes fired by the master neuron (lower trace). (b) Spike fired by the master neuron and anticipated spike fired by the slave neuron.

bucket brigade circuits (MN3004). A function generator with white noise output capabilities (HP33120A) was used to excite both electronic neurons. The signals were acquired using LabView and National Instruments DAQ $6025 \mathrm{E}$ data acquisition board.

Similar electronic neurons have been implemented in [6], where it was shown that their behavior is very similar to that of biological neurons: When interfaced to biological neurons, hybrid circuits could be formed which could function normally (with the electronic neurons taking the place of missing or damaged biological neurons).

In our case, for an appropriate value of the coupling resistance $R_{C}$, we observe that, after a transient, the master and slave electronic neurons synchronize in such a way that the slave neuron anticipates the fires of the master neuron by a time interval approximately equal to the delay time $\tau$ of the feedback mechanism. Figure 5(a) shows a typical spike train, and Fig. 5(b) displays in detail a single spike [7]. We observe that, as in the numerical simulations, the firings of the master and the slave neurons start at about the same time: Anticipation begins during the rising of the peak and it vanishes when the neurons are in the unexcited state. Without coupling and feedback $\left(R_{C}=R_{D}=0\right)$, the neurons fire pulses which are, in general, desynchronized (due to the small mismatch between the circuits).

In summary, we have studied the regime of anticipated synchronization in coupled systems exhibiting neuronaltype excitable behavior, when they are driven by external aperiodic forcing. We have shown that, under appropriate conditions, the slave system can anticipate the random spikes of the master system, in spite of the fact that, since the coupled systems are nonautonomous, the anticipated synchronization manifold is not a solution of the equations. We have modeled the coupled neurons with the FitzHugh-Nagumo and a modified Hodgkin-Huxley model. The FitzHugh-Nagumo model was also implemented in analog hardware. We have considered different types of random forcing, showing that the anticipation phenomenon is robust. Our results show that nonlinearity, noise, and delayed feedback might conspire to produce new interesting and unexpected phenomena, and we hope that our findings will stimulate the search for anticipated synchronization in biological systems.

The work is supported by MCyT (Spain) and FEDER, Projects No. BFM2001-0341-C02-01 and No. BMF20001108. C. Massoller acknowledges partial support from the UIB.

[1] S. Boccalelli et al., Phys. Rep. 366, 1 (2002).

[2] H. U. Voss, Phys. Rev. E 61, 5115 (2000); Phys. Rev. E 64, 039904(E) (2001); Phys. Rev. Lett. 87, 014102 (2001).

[3] A. Longtin, A. Bulsara, and F. Moss, Phys. Rev. Lett. 67, 656 (1991); J. J. Collins, C. C. Chow, and T. T. Imhoff, Phys. Rev. E 52, R3321 (1995); Nature (London) 376, 236 (1995); D. R. Chialvo, A. Longtin, and J. MüllerGerking, Phys. Rev. E 55, 1798 (1997).

[4] L. Glass and M. C. Mackey, From Clocks to Chaos: The Rhythms of Life (Princeton University Press, Princeton, NJ, 1988).

[5] H. A. Braun et al., Int. J. Bifurcation Chaos Appl. Sci. Eng. 8, 881 (1998); U. Feudelet et al., Chaos 10, 231 (2000); W. Braun et al., Phys. Rev. E 62, 6352 (2000).

[6] R. C. Elson et al., Phys. Rev. Lett. 81, 5692 (1998).

[7] Time traces of experimental spike trains as well as a detailed description of the electronic circuit can be found in the web page: http://www.imedea.uib.es/ claudio. 